\title{
Search for Anomalous Single Top Production at HERA
}

Stefano Antonelli on behalf $\mathrm{H} 1$ and ZEUS Coll.

University of Bologna \& INFN

Madrid, DIS09 


\section{HERA performances}

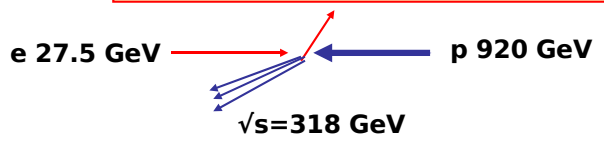

Lumi integrated by experiments:

HERA I

$e+p \sim 100 \mathrm{pb}-1$

e-p 15 pb-1

HERA II (polarisation

$30 \%)$

$\mathrm{e}+\mathrm{p} \sim 200 \mathrm{pb}-1$

e-p $\sim 200$ pb-1

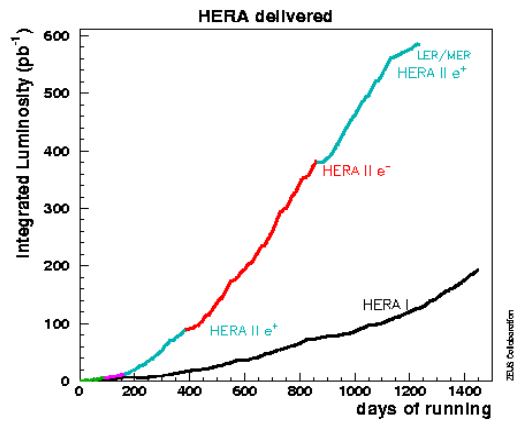

\section{HERA running up to summer 2007}

Results on anomalous single top production presented

H1 $474 p b^{-1}$ full $e^{ \pm} p$ data sample, HERAI+II published DESY-09-050 ZEUS $277 p b^{-1}$ (HERAll prelim.) $+120 p b^{-1}$ (HERAI, publ. PLB 559 (2003) 153) 


\section{Anomalous Single Top Production}

At HERA single top search is related to events with high- $p_{T}$ leptons and high missing $p_{T}$ but also the hadronic decay of $W$ can be exploited.
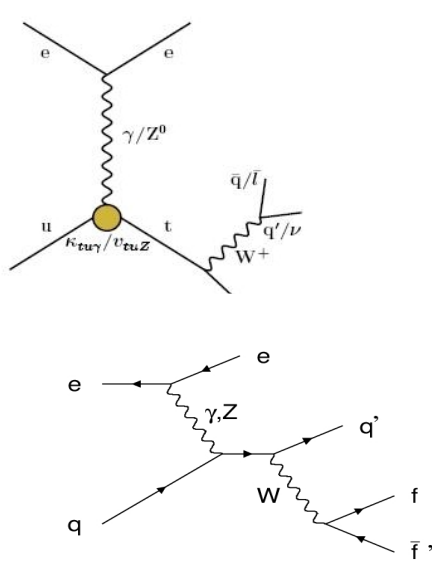

Anomalous single top production (BSM) proceeds via FCNC with a coupling $\kappa_{t u \gamma}$.

$\mathcal{L}=\frac{e e_{u}}{\Lambda} \bar{t} \sigma_{\mu \nu} q^{\nu} \kappa_{\gamma} u A^{\mu}$

couplings to $c$ and $Z$ are neglected

In SM, events with isolated lepton are mainly due to $W$ production $(\sim 1.2 p b)$. 


\section{Anomalous Single Top Production}

At HERA single top search is related to events with high- $p_{T}$ leptons and high missing $p_{T}$ but also the hadronic decay of $W$ can be exploited.
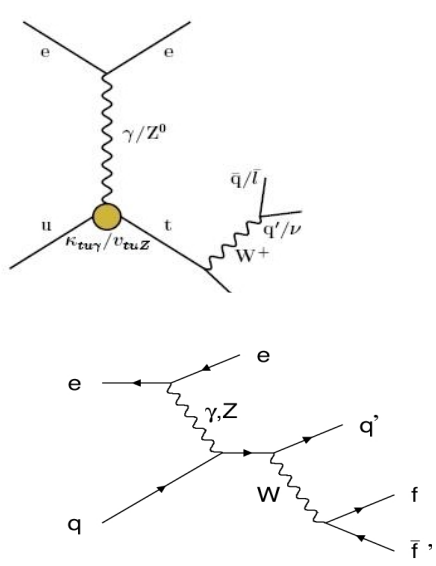

Anomalous single top production (BSM) proceeds via FCNC with a coupling $\kappa_{t u \gamma}$.

$$
\mathcal{L}=\frac{e e_{u}}{\Lambda} \bar{t} \sigma_{\mu \nu} q^{\nu} \kappa_{\gamma} u A^{\mu}
$$

couplings to $c$ and $Z$ are neglected
In SM, events with isolated lepton are mainly due to $W$ production $(\sim 1.2 p b)$.

HERA experiments are sensitive to $u$ (valence quark) and anomalous $\gamma$ exchange 


\section{H1 Anomalous Single Top Search}

Result published: DESY-09-050

Full data sample collected by $\mathrm{H} 1$ at HERA: integrated luminosity $474 p b^{-1}$

Search for anomalous top production looking at muon, electron and hadron channels

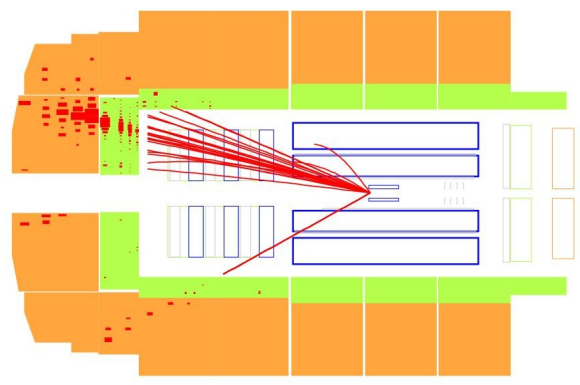

Multivariate discriminant method based on a neural network used to differentiate top quark production from SM background 


\section{Leptonic Channel - H1}

\section{Leptonic Channel}

(based on the isolated

lepton analysis

arXiv:0901.0488)

Leptons isolated from jets and other tracks in the event and:

$$
p_{T, \text { miss }}>12 \mathrm{GeV}
$$

$p_{T}$ of the leptons $>10 \mathrm{GeV}$

polar angle $0.1<\theta<2.4$ rad

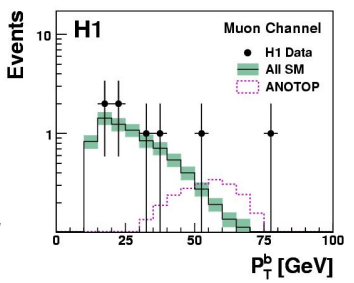

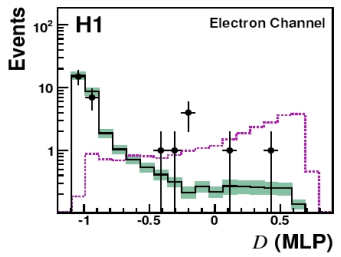

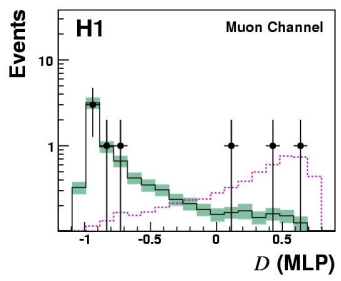

The data are in overall agreement with the SM pred. A slight excess of data in the signal region is visible. 


\section{Hadronic channel - H1}

\section{Hadronic Channel}

Events with at least three jets in $-0.5<\eta<2.5$

Jets ordered in transverse momenta:

$p_{T, 1}>40 \mathrm{GeV}, p_{T, 2}>$ $30 \mathrm{GeV}, p_{T, 3}>15 \mathrm{GeV}$

Cut on the scalar sum of the jet transverse momenta

$$
\sum p_{T, j e t}>110 \mathrm{GeV}
$$
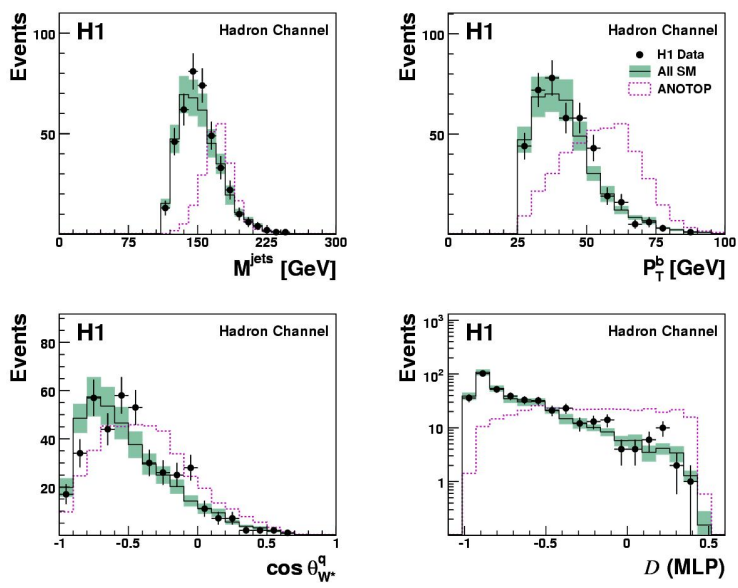

Good overall agreement between data and SM prediction. 


\section{H1 Limit Setting}

No significant excess is seen $\Rightarrow$ limits on the single top production have been set

\begin{tabular}{|c|cc|c|c|}
\hline \multicolumn{6}{|c|}{ H1 Search for Single Top Production $\mathbf{e}^{ \pm} \mathbf{p}, \mathbf{4 7 4} \mathbf{p b}^{-1}$} \\
\hline \hline & \multicolumn{4}{|c|}{ Upper Limit at 95\% CL } \\
\hline Channel & $\begin{array}{c}\sigma(e p \rightarrow t X, \sqrt{s}=319 \mathrm{GeV}) \\
\text { Observed[pb] }\end{array}$ & $\kappa_{\text {tu } \gamma}$ & $\begin{array}{c}\mathcal{B}(t \rightarrow u \gamma) \\
{[\%]}\end{array}$ \\
\hline Electron & 0.40 & 0.24 & $0.21-0.23$ & $0.82-1.02$ \\
Muon & 0.30 & 0.22 & $0.18-0.20$ & $0.61-0.76$ \\
Electron+Muon & 0.27 & 0.15 & $0.17-0.19$ & $0.55-0.69$ \\
Hadronic & 0.42 & 0.27 & $0.21-0.24$ & $0.86-1.07$ \\
Combined & 0.25 & 0.12 & $0.16-0.18$ & $0.51-0.64$ \\
\hline
\end{tabular}

The limits are larger than the expected ones evaluated with a toy model assuming a pure SM scenario, since a slight excess of events, compatible with the signal, have been observed.

$\mathrm{H} 1$ has included NLO corrections to the signal cross section in the coupling limit evaluation.

$\Lambda=M_{\text {top }}=175 \mathrm{GeV}$; left values of $\kappa$ and $B r$ assume $\Lambda=M_{\text {top }}=170 \mathrm{GeV}$. Systematic errors are taken into account. 


\section{ZEUS Anomalous Single Top Search}

Muon Channel:

$$
\begin{gathered}
\left|Z_{v t x}\right|<30 \mathrm{~cm} \\
E-p_{Z}<70 \mathrm{GeV} \\
p_{T, \text { miss }}>10 \mathrm{GeV}
\end{gathered}
$$

Muon Candidate:

$$
p_{T}>8 \mathrm{GeV}
$$

track from primary vertex and isolated from other tracks in the event

\section{Electron Channel}

$$
\begin{gathered}
\left|Z_{v t x}\right|<30 \mathrm{~cm} \\
5<E-p_{Z}<50 \mathrm{GeV}
\end{gathered}
$$$$
p_{T, \text { miss }}>12 \mathrm{GeV}
$$

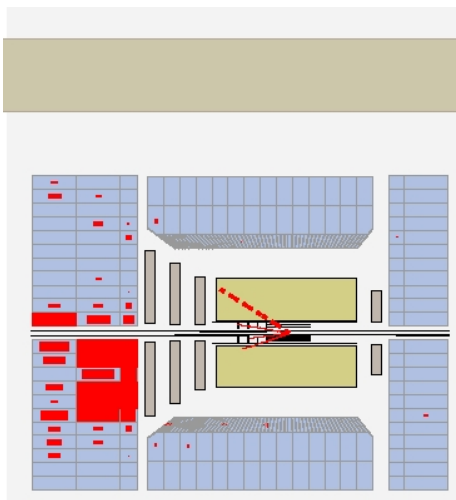

\section{Electron Candidate:}

$$
p_{T}>10 \mathrm{GeV}
$$

track from primary vertex and isolated from other tracks in the event

$0.1<$ acoplanarity $<\pi-0.1$ rad 


\section{Muonic Channel - ZEUS}

ZEUS
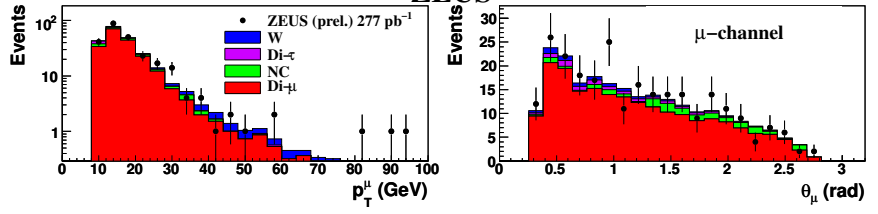

Acceptable
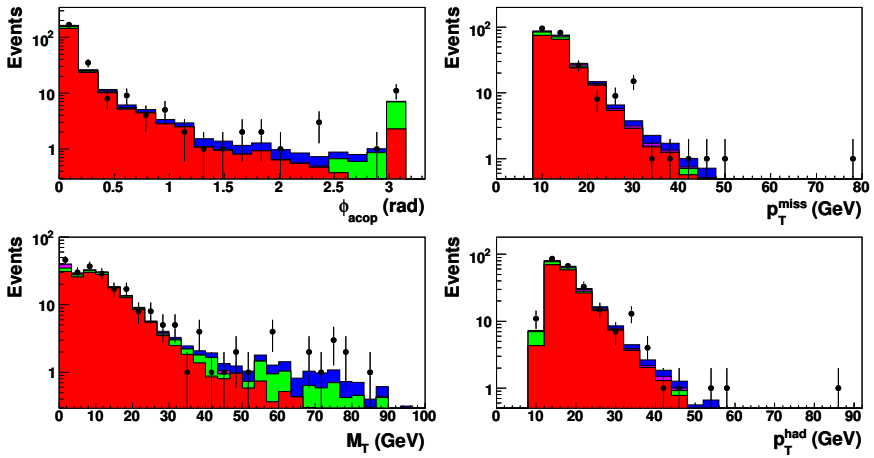

agreement between data MonteCarlo.

Main background: di-muon production.

$W$ contribution visible at high transverse mass $M_{T}$. 


\section{Electronic Channel - ZEUS}

ZEUS
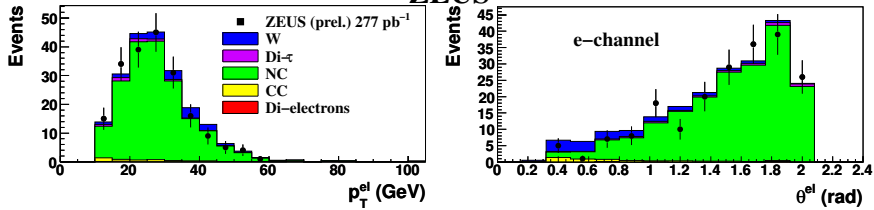

Good agreement between data MonteCarlo.
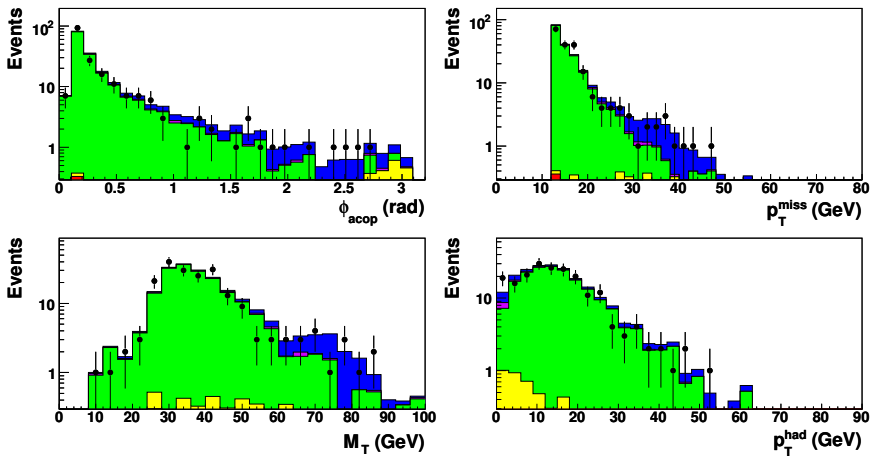

Main background: Neutral Current.

$W$ contribution visible at high- $p_{T}$ and high transverse mass $M_{T}$. 


\section{Final Cuts - ZEUS}

For the final selection a cut on $p_{T, \text { had }}>40 \mathrm{GeV}$ has been required

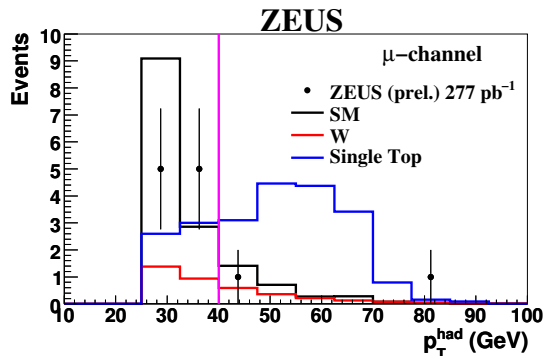

\section{Muon Channel}

Acoplanarity $>0.05$ rad

Events with more than one isolated muon have been rejected

\section{Electron Channel}

Acoplanarity $>0.15 \mathrm{rad}$

$p_{T, \text { miss }}>15 . \mathrm{GeV}$

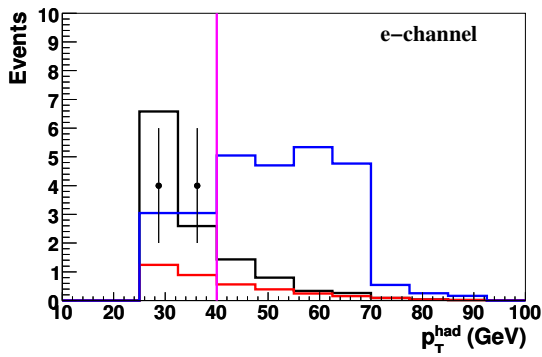

Good agreement between data-MC; no discrepancy at high $\mathrm{p}_{\mathrm{T}, \mathrm{had}}$ 


\section{ZEUS Limit Setting}

\section{Single Top Selection}

\begin{tabular}{ccccc}
\hline & $N_{\text {obs }}$ & $N_{\text {pred }}$ & $W[\%]$ & Eff. $\times$ Br. \\
\hline Muon Channel 04-05 $e^{-} p$ & 1 & $1.5 \pm 0.4$ & 47 & 0.026 \\
Muon Channel 06-07 $e^{+} p$ & 1 & $1.4 \pm 0.4$ & 50 & 0.026 \\
\hline Electron Channel 04-05 $e^{-} p$ & 0 & $2.1 \pm 0.6$ & 38 & 0.033 \\
Electron Channel 06-07 $e^{+} p$ & 0 & $0.9 \pm 0.3$ & 78 & 0.033 \\
\hline
\end{tabular}

These results have been converted into limit on the signal cross section using a Bayesian approach. Assuming a constant prior on the signal cross section. The following upper limit on the cross section has been obtained:

$$
\sigma<0.23 p b(95 \% \text { C.L. })
$$

The limit on the cross section has been converted into a limit on the coupling $\kappa_{t u \gamma}$

$$
\kappa_{\text {tur }}<0.17\left(0.16-0.18, M_{\text {top }}=171.2 \pm 2.1 \mathrm{GeV}\right)
$$

The result has been combined with the HERA I limit (using only samples at $\sqrt{s}=318 \mathrm{GeV}$ ) for a total integrated luminosity of $359 \mathrm{pb}^{-1}$ :

$$
\sigma<0.13 p b, \kappa_{\text {tu }}<0.13(\operatorname{Br}(t \rightarrow u-\gamma)<0.34 \%)
$$




\section{Comparison of HERA Limits with Other Experiments}

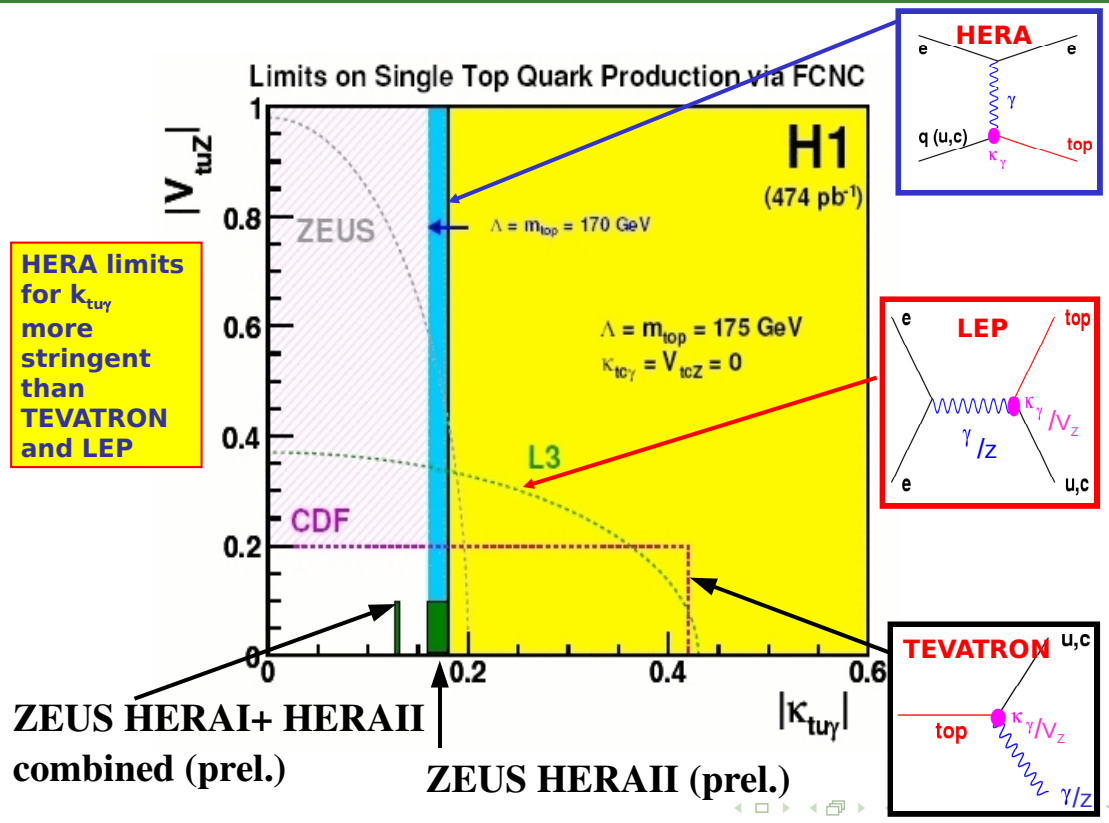




\section{Conclusions}

H1 has published analysis on anomalous single top production exploiting full HERAI + HERAll statistics (DESY-09-050), $\sigma<0.25 p b, \kappa_{\text {tu }}<0.18$

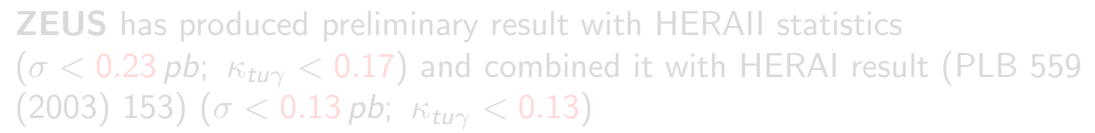

The study on single top production is a good example of complementarity between different colliders

For the process involving a $u$ - top transition mediated by photon, HERA constraints are the best to date 


\section{Conclusions}

H1 has published analysis on anomalous single top production exploiting full HERAI + HERAll statistics (DESY-09-050), $\sigma<0.25 p b, \kappa_{t u \gamma}<0.18$

ZEUS has produced preliminary result with HERAII statistics $\left(\sigma<0.23 p b ; \kappa_{t u \gamma}<0.17\right)$ and combined it with HERAI result (PLB 559 (2003) 153) $\left(\sigma<0.13 p b ; \kappa_{\text {tu }}<0.13\right)$

The study on single top production is a good example of complementarity between different colliders 


\section{Conclusions}

H1 has published analysis on anomalous single top production exploiting full HERAI + HERAll statistics (DESY-09-050), $\sigma<0.25 p b, \kappa_{t u \gamma}<0.18$

ZEUS has produced preliminary result with HERAII statistics $\left(\sigma<0.23 \mathrm{pb} ; \kappa_{\text {tu }}<0.17\right)$ and combined it with HERAI result (PLB 559 (2003) 153) $\left(\sigma<0.13 p b ; \kappa_{\text {tu }}<0.13\right)$

The study on single top production is a good example of complementarity between different colliders 


\section{Conclusions}

H1 has published analysis on anomalous single top production exploiting full HERAI + HERAll statistics (DESY-09-050), $\sigma<0.25 p b, \kappa_{t u \gamma}<0.18$

ZEUS has produced preliminary result with HERAII statistics $\left(\sigma<0.23 \mathrm{pb} ; \kappa_{\text {tu }}<0.17\right)$ and combined it with HERAI result (PLB 559 (2003) 153) $\left(\sigma<0.13 p b ; \kappa_{\text {tu }}<0.13\right)$

The study on single top production is a good example of complementarity between different colliders

For the process involving a $u-$ top transition mediated by photon, HERA constraints are the best to date 


\section{Backup}
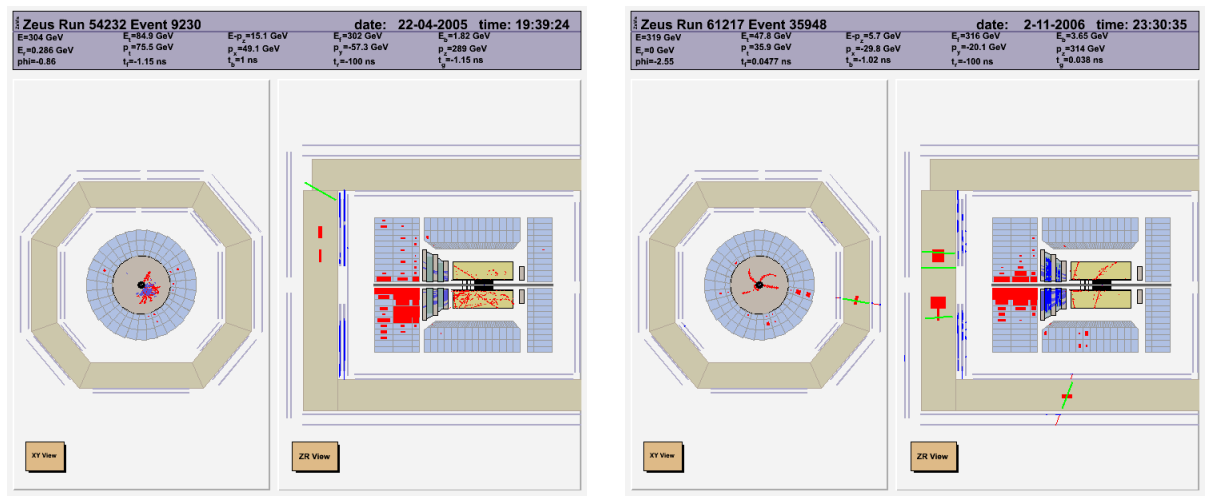

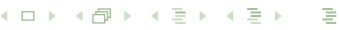




\section{Backup}

Cuts optimization $\Rightarrow$ minimizing the cross section upper limit $\left(\sigma_{\text {lim }}\right)$ assuming a pure SM scenario:

$$
\left\langle\sigma_{\text {lim }}\right\rangle=\frac{\left\langle N_{s i g}^{l i m}\right\rangle}{\epsilon \mathcal{L}}
$$

where $\epsilon$ is the signal efficiency, $\mathcal{L}$ is the integrated luminosity and $\left\langle N_{\text {sig }}^{\text {lim }}\right\rangle$ is the limit on the number of signal events averaged over the possible observed events;

$$
\left\langle N_{\text {sig }}^{l i m}\right\rangle=\sum_{N_{o b s}=0}^{\infty} P\left(N_{o b s}, N_{b k g}\right) \cdot N_{s i g}^{\lim }\left(N_{o b s}, N_{b k g}\right),
$$

$N_{s i g}^{\lim }\left(N_{o b s}, N_{b k g}\right)$ being the limit on the number of the signal events at $95 \%$ C.L. 


\section{Backup}

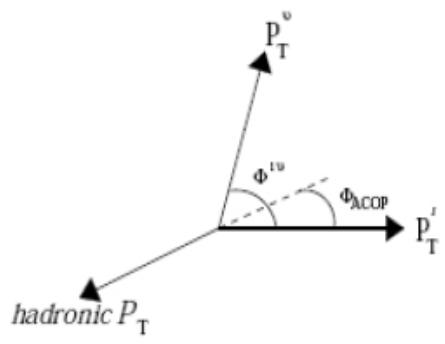

Acoplanarity is the angle in the transverse plane between the lepton and the vector balancing the hadronic $p_{T}$ 